\title{
MedienPädagogik
}

Zeitschrift für Theorie und Praxis der Medienbildung

www.medienpaed.com

ISSN 1424-3636

Jahrbuch Medienpädagogik 17:

Lernen mit und über Medien in einer digitalen Welt

Herausgegeben von Klaus Rummler, Ilka Koppel, Sandra Aßmann,

Patrick Bettinger und Karsten D. Wolf

\section{Bildung in der digitalen Welt: Über Wirkungsannahmen und die soziale Konstruktion des Digitalen}

\author{
Michael Kerres
}

\section{Zusammenfassung}

Die Diskussion über die Digitalisierung in der Bildung beschäftigt sich mit der Frage, was für die Zukunft gelehrt und gelernt werden soll, und ist dabei mit der Unsicherheit von Zukunftsentwürfen konfrontiert. Auf der Basis gesellschaftlicher Verständigungsprozesse weisen Kompetenzlisten aus, was für die Zukunft wichtig ist und gelernt werden sollte. Sie sind vielfach so angelegt, dass sie ihre Zukunftsentwürfe verstecken, und so der weiteren Verständigung entziehen. Der Beitrag kontrastiert kulturtheoretische Überlegungen zur digitalen Epoche mit den oftmals impliziten Annahmen vorliegender Entwürfe, die regelmässig auf einen Technikdeterminismus verweisen. Sie schreiben der Technik die entscheidende Wirkung auf gesellschaftliche Entwicklung zu und verdecken die Gestaltungsoptionen in der handelnden Auseinandersetzung mit der amorphen Digitaltechnik. Auf dem Hintergrund bildungstheoretischer Überlegungen wird das Verständnis von Bildung in der digitalen Welt vorgestellt und in Beziehung gesetzt zu den generischen und domänenspezifischen Kompetenzen zu ihrer gestaltenden Teilhabe. 


\title{
Bildung in a Digital World: On impact assumptions and the social construction of future
}

\begin{abstract}
The discussion about digital education refers to the question what should be taught and learned for the future. Since it has to deal with the essential uncertainty of drafting a future, lists of competencies are developed that declare which competences seem important for coping with future challenges. These lists are based upon assumptions about the future. However, they hide these assumptions and, thus, withdraw them from further discussions. The article contrasts theories from cultural studies about the «next society» with the frequently implicit assumptions of the existing drafts. Regularly, they refer to the idea of technology as determining the development of society which hides the design options in the process of negotiating the future of the - largely amorphous - digital technology. On the background of theories of Bildung the concept of «education in a digital world» is introduced and related to the generic and domain specific competencies necessary for participating in creating the future.
\end{abstract}

\section{Wirkungen des Digitalen}

Blicken wir auf den Diskurs über das Digitale im Kontext von Bildung in den letzten Jahrzehnten, dann fällt auf, dass sich dieser nur wenig verändert hat. Die jeweiligen Techniken, die in der Öffentlichkeit Aufmerksamkeit erhalten, mögen sich verändern; ihre Bewertung folgt jedoch einem stets ähnlichen Muster: Auf der einen Seite finden sich die Befürworter, die mit der Digitalisierung positive Entwicklungen verbinden. Sie sprechen von Bildungsinnovationen oder -revolutionen, von überlegenen Lernleistungen und drängen auf eine intensivere Beschäftigung mit diesen Möglichkeiten, um die Chancen der Digitalisierung - auch im internationalen Wettbewerb - einzulösen (s. etwa Dräger und Müller-Eiselt 2015).

Zugleich wird von einer Gegenposition regelmässig das Absinken der Lern- und Bildungsstandards befürchtet. Es wird auf zu geringe körperliche Aktivität bei langem Computerkonsum verwiesen und damit 
verbundene Suchtgefahren. Problematisiert wird insbesondere ein zu früher und zu extensiver Einsatz des Computers in Kindergarten und Schule. Dabei wird häufig auf «die Hirnforschung» verwiesen, die die negativen Wirkungen einer Bildschirm- und Computernutzung belege (s. etwa Spitzer 2005).

Ein grosser Teil der internationalen Forschung zu Educational Techno$\log _{Y}$ ist genau dieser Kontroverse gewidmet. Die typische Anlage entsprechend quasi-experimenteller Forschungsdesigns vergleicht das Lernen mit digitalen Medien und traditionellem Unterricht. Seit den ersten Arbeiten von Kulik (1980) liegen Metaanalysen vor (s.a. Tamim u.a. 2011), die auf der Grundlage der Vielzahl von aggregierten Einzelstudien einen vergleichsweise geringen Effekt digitaler Medien auf Lernergebnisse zeigen: Im Vergleich zu traditionellem Unterricht tragen digitale Medien nicht grundsätzlich dazu bei, dass das Lernen mit digitalen Medien zu substantiell höheren, aber auch nicht zu niedrigeren Lernleistungen führt (s.a. Zierer 2019).

Dabei wird seit Anbeginn dieser Forschung problematisiert, inwiefern solche Vergleichsstudien aussagekräftig sind (G. Salomon und Clark 1977; Clark 1983): Was bedeutet «traditioneller Unterricht» und lässt sich mit diesem Label tatsächlich der Referenzwert einer Kontrollgruppe bestimmen? Sind E-Learning, E-Books, Virtual Classrooms, Augmented Reality oder MOOCs tatsächlich Treatments - oder doch nur die Verpackung für den eigentlichen Wirkstoff (Bernard u.a. 2014; 2018)?

Besonders auffallend ist, dass diese Studien so angelegt sind, dass der jeweiligen Technik, einer Hardware oder einer Software, die entscheidende Wirkung auf das Lernen zugeschrieben wird: Der Forschung ist ganz selbstverständlich die Annahme hinterlegt, es sei die jeweilige Technik selbst, die einen Lernerfolg beeinflussen würde, nicht aber Faktoren wie die in dem Medium realisierte didaktische Konzeption, nicht die Qualität der Interaktion der Lernenden mit einer Technik, nicht die Passung der in der Technik umgesetzten Konzepte zu Lehrzielen oder weiteren Merkmalen des didaktischen Feldes, wie sie als Erfolgsbedingungen in der Mediendidaktik diskutiert werden (vgl. Kerres 2018).

Diese Vergleichsstudien reagieren auf die Debatte über das Pro und Contra der Bildungstechnologie und scheinen damit zur gesellschaftlichen 
Diskussion beizutragen. Die Studienlage kann die Überlegenheit einer Digitalisierung jedoch nicht überzeugend belegen. Zugleich sind die Forschungsdesigns solcher Vergleichsstudien grundsätzlich infrage gestellt worden. Auch die auf Grundlage solcher Studien erstellten Metaanalysen helfen dann nur bedingt weiter. Insofern wird man Studien hinterfragen wollen, die «die» Wirkung der Technik auf das Lernen zum Gegenstand haben.

Die Mediendidaktik beschäftigt dagegen seit Langem die Frage, wodurch eigentlich «Wirkungen» beim Lernen mit Medien entstehen. Dabei stehen eine Reihe von Sichtweiten im Raum, die sich nicht wechselseitig ausschliessen:

- Sind Medien (wie «das Buch», «das Fernsehen» oder «das Internet») nur unterschiedliche «Transporter»(mere vehicles), die den Lernprozess nicht beeinflussen (Clark 1994)?

- Entscheidet die didaktische Konzeption eines Mediums, das instructional design, über die Wirksamkeit eines mediengestützten Lernangebotes (Kozma 1991)?

- Kommt die Wirkung durch die Qualität der Supplantation zustande, wie Salomon (1972) es formulierte, wonach eine bestimmte Aufbereitung von Information - in Relation zur kognitiven Struktur des Lernenden mentale Aneignungsprozesse unterstützt?

- Wirken Medien dadurch, dass sie Routen für Kommunikation kreieren und damit gesellschaftliche Machtverhältnisse etablieren, wie es ursprünglich Harrold Innis aufzeigte (vgl. Heyer 2003)?

- Erzeugt das Medium Realität durch die Art der Nutzung (in der eine Gratifikation entsteht, vgl. Lin 1996) und durch Zuschreibungen der Rezipienten (Schmidt 1996; aber auch: 2003)?

- Entstehen Wirkungen durch bestimmte rhetorische Figuren, Begründungsmuster und Metaphern, in der öffentlichen Rede über Medien (M. Kerres 2003; 2017)?

- Sind Wirkungen des Mediums bereits in das Medium und in die $\mathrm{Me}$ dienwahl eingeschrieben: Schreibt das Medium an den Inhalten mit (vgl. etwa die Diskussion in der Linguistik bei Siever, Schlobinski, und Runkehl 2009)? 
Für jede dieser Positionen lassen sich Bezüge zu Ansätzen in der Medientheorie und Mediensoziologie, der Kommunikationswissenschaft und Medienpädagogik herstellen. Nur: für die Annahme einer unmittelbaren «Wirkung» bestimmter Medientechniken an sich auf Lernen lässt sich hier kaum eine Begründung finden. Es lässt sich aufzeigen: Medien haben Wirkungen, indem sie Wege für Kommunikation schaffen und definieren, wer mit wem wie in welcher Qualität kommunizieren kann, und damit Kommunikationsstrukturen definieren. Gesellschaftliche Entwicklung lässt sich entlang der Epochen beschreiben, in denen jeweils unterschiedliche Medien dominieren - in den Übergängen von der Stammesgesellschaft (mit der Gebundenheit an den Einzelnen in der Mündlichkeit) über die antike Gesellschaft (mit der Schriftlichkeit als kulturelles Gedächtnis) zur modernen Gesellschaft (mit der Verbreitung des Buchdrucks) und zur globalen Kommunikation (über elektronische und digitale Medien), mit der Umbrüche und Machtverschiebungen einhergehen (Jäckel 2010; Baecker 2017).

Diesen langfristigen, epochalen Einschnitten steht die Indifferenz gegenüber, mit der das Bildungssystem bislang auf die neuen Technologien reagiert. Die Verfügbarkeit digitaler Medien wirkt sich zunächst nur marginal auf didaktische Konzepte, auf die Arbeit in Bildungseinrichtungen und das Verhalten von Lehrenden und Lernenden aus. Digitale Medien können in Konzepten des projekt- und problemorientieren Lernens Einsatz finden, genauso wie sie Formate eines Frontalunterrichts anreichern. Sie werden kaum Veränderung von Unterrichts- resp. Lehrverhalten nach sich ziehen. In der Verunsicherung, die durch neue Anforderungen entsteht, wird vielmehr auf bekannte Handlungsmuster zurückgegriffen: Der Beamer im Seminarraum ist vermutlich deswegen so erfolgreich, weil er jahrzehntelang stabilisierte Routinen des Lernens und Lehrens in der Präsentation und der frontalen Unterweisung stärkt. Es erscheint plausibel, von der Beharrlichkeit überlernter Handlungsroutinen der Welterschliessung auszugehen: In der Nutzung digitaler Werkzeuge und Medien werden über Jahrzehnte aufgebaute Verhaltensweisen eingebracht und es bedarf recht weit angelegter Ansätze der Organisations- resp. Schulentwicklung, um systemische Veränderungen in den Handlungspraktiken der Akteure zu erreichen (van Ackeren u.a. 2017). 
Es war das massgebliche Verdienst von Paul Heimann (1976) die Entscheidung für eine didaktische Methode einerseits und die Wahl eines Mediums andererseits als separate Felder didaktischer Planung zu beschreiben: Methoden- und Medienentscheidungen stehen zwar in einem Verhältnis der Interdependenz (d.h. die Entscheidung für eine Methode hat Implikationen für die Medienwahl), sie sind aber systematisch als unabhängig zu verstehen: Ich kann Projektunterricht mit ausgedruckten Materialien, auf einem E-Book oder im Internet zum Download anbieten - ohne dass diese Medienwahl die Frage des Projektunterrichts grundlegend beeinflusst. Ich sollte mich allerdings für genau das Medium entscheiden, das u.a. dem Lehrziel, der Methode oder anderen Rahmenbedingungen optimal zuträgt.

Reformpädagogisch motivierte Weiterbildungsansätze (gerade in der Fortbildung für Lehrpersonen) basieren dagegen regelmässig auf der Annahme, dass die Digitalisierung Schule verändert oder sich Schule wird ändern müssen (s. etwa Krommer u.a. 2019). Manchmal überwiegt die Aussage, dass das Digitale die Schule verändern «wird», manchmal überwiegt die Aussage, dass durch das Digitale sich Schule «wird ändern müssen». Es wird ein «zeitgemässer» Unterricht beschrieben und wie Unterricht im digitalen Zeitalter aussehen wird. Vielfach verbunden ist damit die Annahme, dass die digitale Technik zu neuen Unterrichtsformen führen wird: Verbreitet ist etwa die Annahme, dass sich die Rolle der Lehrpersonen ändert und vom Vermittler zum Coach entwickelt, dass die Lernenden künftig selbstgesteuert statt fremdgesteuert lernen werden usw.

Der appellative Charakter dieser Aussagen ist auffallend: Der Wunsch nach Reformen wird auf dem Hintergrund des Digitalen begründet und gleichzeitig wird dem Digitalen die Wirkmächtigkeit zugeschrieben, diese Hoffnungen Wirklichkeit werden zu lassen. ${ }^{1}$ Aus den bisherigen Überlegungen wäre abzuleiten: Schule muss sich auf dem Hintergrund veränderter gesellschaftlicher Rahmenbedingungen immer hinterfragen; dazu gehört auch die Digitalisierung. Allerdings ist das Verhältnis von Digitalisierung und den schulischen Inhalten und Lernformen deutlich vielschichtiger: Die Einführung der Computer in Schulen trägt keinesfalls zu

1 Damit soll im Übrigen die Bedeutung entsprechender Bemühungen nicht grundsätzlich angezweifelt werden; es geht hier nur um die Argumentationslogik ihrer Begründung. 
bestimmten Veränderungen des Lehrens und Lernens bei. Sind diese erwünscht, müssen sie aktiv herbeigeführt werden. Dabei können digitale Medien eine wichtige Rolle spielen.

Das Besondere der Diskussion besteht in der Gleichzeitigkeit einer Wirksamkeit und Unwirksamkeit der digitalen Medien im Kontext der Bildung. Das Printbuch als digitales Produkt auf einem E-Book Lesegerät ausgeliefert, ändert mein Leseempfinden eher wenig, auch wenn eine Reihe von Vor- und Nachteilen damit einhergehen, die dazu führen, dass ich manchmal ein gedrucktes Buch bevorzuge, manchmal ein digitales Produkt. Gleichzeitig verändert die Verfügbarkeit digitaler Bücher den gesamten Produktionsprozess - und letztlich möglicherweise die Bedeutung des Buches in einer Kultur. Vermutlich sind die «starken» Wirkungen des Digitalen, wie sie etwa Baecker (2007; 2018) in der «nächsten Gesellschaft» beschrieben hat, vor allem epochaler Natur, d.h. erst über Jahrhunderte erkennbar; kurzfristig ist für die pädagogische Arbeit dagegen mit eher «schwachen» Wirkungen zu rechnen.

\section{Gestaltungsoptionen der digitalen Epoche}

Es wurde deutlich, dass die Debatte über die Wirkungen des Digitalen ebenso wie die Vergleichsstudien annehmen, die Technik selbst würde das Lernen und die Lernqualität verändern und aus der Kenntnis der Technik würden sich unmittelbar Konsequenzen, etwa für zu erwerbende Kompetenzen, ableiten lassen. Eine sozio-konstruktivistische Position sieht die Wirkungen des Digitalen dagegen als Ergebnis sozialer Aushandlungsprozesse, die dann Realitäten hervorbringen. Sie basieren auf der individuellen Konstruktion von Bedeutungen in der Auseinandersetzung mit Technik. Eine Technik eröffnet unterschiedliche Routen, wie ihre Verwendung gesellschaftlich organisiert wird: Bei der Erfindung des Automobils war keineswegs offensichtlich, dass es einmal eine Strassenverkehrsordnung, normierte Strassenschilder, Führerscheine und Strafpunkte für falsches Fahren geben würde. Betrachten wir den Rahmen, den der Übergang zur digitalen Epoche bietet. 


\section{Kontrolle neu denken}

Digitale Computer funktionieren auf der Basis von Algorithmen, die eine endliche Folge von Schritten beschreiben, mit der sich eine Klasse von Problemen zuverlässig lösen lässt. Die Arbeitsweise des einzelnen Computers, gesteuert von Algorithmen, die von Menschen programmiert wurden, scheint - bei Verständnis der Funktionsweise digitaler Technik und Automaten - grundsätzlich nachvollziehbar, kontrollierbar und vorhersagbar. Auf der Ebene der vernetzten globalen Systeme verliert sich diese Gewissheit. Wir sind - als Privatpersonen wie auch als Entscheiderinnen und Entscheider in Funktionen - zunehmend darauf angewiesen, Informationen aufzunehmen und Entscheidungen zu treffen, ohne die Quelle und Qualität von Informationen zuverlässig prüfen und ihr Entstehen bewerten zu können. Es geht nach Baecker (2017) nicht mehr nur um den in früheren Epochen entwickelten - Referenzüberschuss von Sprache, den Symbolüberschuss von Schrift und den Kritiküberschuss des Buchdrucks. In der digitalen Epoche wird der Kontrollüberschuss zu bewältigen sein, der entsteht, weil sich die Computer mit ihrem Gedächtnis an gesellschaftlicher Kommunikation beteiligen. Menschen können die Prozesse der im Netzwerk interagierenden digitalen Systeme in ihren Ergebnissen nicht mehr im Einzelnen nachvollziehen (s.a. Algorithmizität bei Stalder 2016). Aus Abzählbarkeit, Berechenbarkeit und Steuerbarkeit des den einzelnen Computer zugrundeliegenden Algorithmus werden Unüberschaubarkeit, Unberechenbarkeit und Unvorhersehbarkeit durch die Datafizierung gesellschaftlicher Funktionsbereiche. Damit muss die «nächste Gesellschaft» umgehen. Verschiedene Autoren beschäftigen sich mit den Herausforderungen dieser neuen Konstellation für Gesellschaft (vgl. Nassehi 2019; West 2019; Allert, Asmussen, und Richter 2018; Ramge und MayerSchönberger 2017; O'Neil 2016; Zuboff 2015).

Die qualitative Differenz, die beim Übergang des Einzelcomputers zum globalen Netzwerk digitaler Systeme (einschliesslich dem «Internet of Things») entsteht, soll an einem Beispiel verdeutlicht werden: Auf Grundlage der Daten, die mein Konsumverhaltens im Internet erzeugt, kann ich Empfehlungen erhalten, die mich auf Bücher, Musik oder Videos hinweisen, die mich interessieren könnten. Ich kann Tipps zu sportlichen Aktivitäten, zur Ernährung oder der Partnerwahl erhalten. Diese Information 
empfinde ich - möglicherweise (!) - als Kontrollgewinn in meiner Lebensführung. Auf gesellschaftlicher Ebene mündet diese Entwicklung jedoch in einen essentiellen Kontrollverlust des Menschen. Auch hier finden wir wieder die Gleichzeitigkeit der Effekte von Digitalisierung (auf unterschiedlichen Ebenen der Analyse): Kontrollgewinn und -verlust findet gleichzeitig statt.

Die Forderung, Autonomie und Mündigkeit in der digitalen Welt, etwa durch Aufklärung und Schulungen, herzustellen, stösst an Grenzen. Denn es ist zu konstatieren, dass eine «digitale Souveränität», wie sie etwa vom Verband der Bayrischen Wirtschaft ${ }^{2}$ gefordert wird und die in der Kategorie des Individuums gedacht ist, in der «nächsten Gesellschaft» schwer zu verorten ist, und der Idee einer Beherrschung von Digitaltechnik verhaftet bleibt, wo wir Stellen erkennen, wo sich eine «Beherrschung» des Digitalen als Illusion erweist. Ein in der Buchepoche verhafteter Blick von Bildung verkennt nach Baecker (2018) den Kontrollüberschuss des Digitalen, der letztlich auf ein neues Verhältnis gesellschaftlicher Akteure hinausläuft. Eine als Aufklärung angelegte Medienerziehung, wie sie bislang üblicherweise verstanden werden, stösst damit an Grenzen: Medienerziehung würde dann auch mit den Implikationen des Kontrollverlustes konfrontieren und die Frage aufwerfen, wie sich die Gesellschaft organisieren kann, um damit umzugehen.

\section{Gestaltungsoptionen wahrnehmen}

Die Genese des Internets zeigt, wie Technik Räume öffnet, die Explorationsbewegungen in Gang setzen, die wiederum technische Entwicklungen motivieren, mit denen sich neue Möglichkeiten ergeben. Gebäude, Geräte und andere technische Artefakte, die von Menschen geschaffen sind, beeinflussen das Handeln von Menschen. Der Schlüsselanhänger im Hotel erinnert(e) den Gast, den Schlüssel an der Rezeption abzugeben. Die Bodenschwelle auf der Strasse zwingt den Autofahrer, die Fahrgeschwindigkeit zu reduzieren. Die Sitzanordnung im Hörsaal erzeugt eine Kommunikationssituation, die Dozierenden und Zuhörenden unterscheidet. Latour

2 Vereinigung der Bayerischen Wirtschaft e.V.. 2018. Digitale Souveränität und Bildung. Münster: Waxmann Verlag. 
(1996) beschreibt, wie Menschen und Nicht-Menschen als gleichwertige Teile eines Netzwerkes fungieren.

Das Protokoll TCP-IP ermöglicht es, Daten zwischen Computern auszutauschen. Anders als eine leitungsvermittelte Kommunikation werden die Daten in Pakete aufgeteilt, in deren "Kopf» der Empfänger eingetragen ist. Über dieses Internet-Protokoll konnten seit etwa Anfang der 1970er z.B. E-Mails versendet (SMTP) oder bereitgestellte Dateien abgerufen werden (FTP). Tim Berners Lee setzte 1990 auf dieser Technik auf, um Forschungsergebnisse aus dem Forschungszentrum CERN in der Schweiz weltweit zugreifbar zu machen. Über das http-Protokoll konnten verlinkte Informationen, die mit der Seitenbeschreibungssprache HTML ausgezeichnet waren, aufgerufen werden, die das "World Wide Web» erzeugten. Dass diese Technik einmal für die Abbildung von Funktionen des Radios, des Fernsehens, der Telefonie oder - im Zuge von Web 2.0 - für ganz neue Anwendungen sozialer Plattformen, für Werbung und den Absatz von Waren genutzt werden würde, war weder den Erfindern des IP- noch des http-Protokolls im Ansatz klar.

Die Besonderheit des Digitalen und hier insbesondere des Internets und seiner Folgetechnologien (wie Smartphone, Smarthome, «Internet of Things») besteht in seiner amorphen Anlage: In der Geschichte der Technik hat es wohl noch nie ein Artefakt gegeben, dessen Nutzung und Nutzen so wenig in die Technikgenese eingeschrieben ist. Das Radiogerät empfängt elektromagnetische Funkwellen, die in auditive Informationen übersetzt werden; andere (sinnhafte) Nutzungsvarianten sind dem Gerät nicht eingeschrieben. Es wurde für genau diesen einen Zweck entwickelt und wird entsprechend verwendet. Und der Hörsaal in seiner starren Anordnung lässt nur wenig Dekonstruktion zu.

Das World Wide Web erzeugt seine Bedeutung dagegen erst in der Art, wie es genutzt wird, und entsprechend verunsichert steht die Gesellschaft vor der Frage: Unterliegt das Internet dem Rundfunkgesetz, dem Telekommunikationsrecht, den Bedingungen des Publikationswesens? Wir können das Internet als ein Informations-, als ein Kommunikationsmedium der Massen- oder Individualkommunikation auffassen. Das Internet emuliert alle bisherigen Medien und hat neuartige Medien - in seiner Nutzung 
- hervorgebracht. Die digitale Technik und das Internet sind im Kern als konstruktivistische Medien zu verstehen, bei der die Wahrnehmung der Nutzenden und ihr Nutzungsverhalten ein Artefakt erst erzeugt (sichtbar z.B. in sozialen Plattformen). Der digitalen Technik sind Spielräume hinterlegt, die durch das Handeln der Akteure Realitäten erzeugen, die zu wiederum neuen technischen Entwicklungen führen.

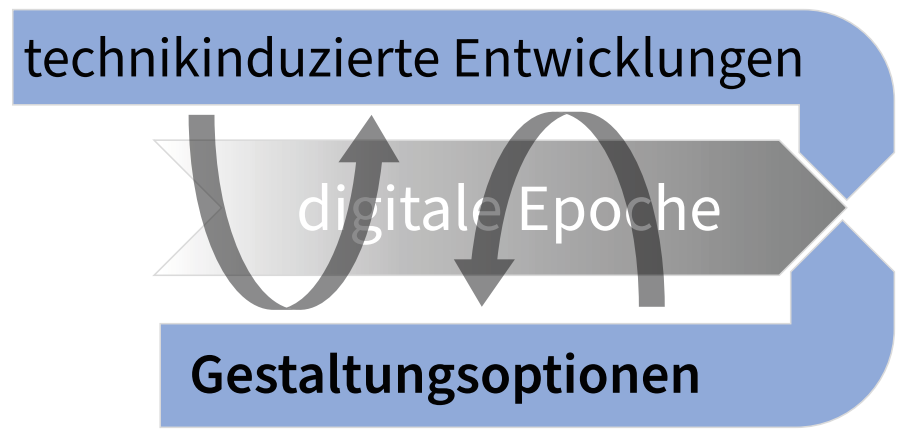

Abb. 1.: Gestaltungsoptionen in der digitalen Epoche.

\section{Bildung auf Ziele ausrichten}

Das Zusammenwirken von technikinduzierten Entwicklungen und der gesellschaftlichen Organisation von Gestaltungsoptionen lässt sich für verschiedene Subsysteme der Gesellschaft aufzeigen: Digitale Technik kann im Ergebnis massgeblich dazu beitragen, eine post-demokratische Gesellschaft zu etablieren, die auf der Ausschöpfung von user-generierten Daten basiert und die weitreichende Steuerung der Person anstrebt (West 2019; Zuboff 2015). Gleichzeitig kann das Handeln der Akteure eine Gesellschaft in der digitalen Epoche entstehen lassen, die auf Werten des Teilens und der Teilhabe an einem «offenen» Wissen einer Kultur basiert, die Austausch und Partizipation als wesentliche Mechanismen des gesellschaftlichen Zusammenlebens anstrebt. Weltweit sind in verschiedenen gesellschaftlichen Kontexten beide Optionen erkennbar und es erscheint damit wenig plausibel zu behaupten, das Internet würde eine bestimmte gesellschaftliche Entwicklung erzeugen. 
Auch im Bildungssystem bestehen Gestaltungsoptionen entlang dieser Logik: Lernen lässt sich im Internet als ein geregelter Prozess implementieren, der auf Grundlage grosser Datenmengen und darauf basierender Algorithmen («learning analytics») optimiert wird (vgl. Ferguson u.a. 2016; Köchling und Riazy 2019). Durch die Überwachung und Auswertung des Lernverhaltens entstehen grosse Datenmengen, die genutzt werden, um eine solche «intelligente» Regulierung des Lernenden zu ermöglichen. Wer den Zugriff auf diese Daten hat, kann den optimalen Regulierungsmechanismus programmieren. Wer diesen Algorithmus besitzt, hat einen Schlüssel zur Steuerung der Menschen und zur Bildung in einer Kultur. Big data und das Internet ebnen damit den Weg zu dem Traum, den der Begründer des Behaviorismus B. F. Skinner (1958) vor langer Zeit formuliert hatte und der auch heute manche Proponenten einer Bildung 4.0 leitet:

«Man kann durch eine elektronische Bildungsakte die erreichten Wissensstadien für jeden Menschen speichern, man kann Auswertungen durchführen, welche Bildungsangebote zu welchen Ergebnissen führen. Besonders herauszustellen ist auch die Individualisierung des Lernens, die durch die Digitalisierung ermöglicht wird.» (Scheer 2017, IT-Gipfel) ${ }^{3}$

Die Gegenposition knüpft an die Idee der Aufklärung an, die Bildung als Selbstregulation auffasst und nicht nur als - möglichst optimierte Aneignung von vorgegebenen Lehr-Lerninhalten. In dieser Tradition ist Bildung immer in Beziehung zur Lebenswelt und zur Gesellschaft zu verstehen und deswegen als «öffentliches Gut» zugänglich zu machen. Das Lernen des Einzelnen bedeutet Teilhabe an dem gewachsenen Wissen und den Erzeugnissen einer Kultur, wie es sich im Internet artikuliert; in dieser Teilhabe entsteht Wissen, und Gesellschaft entwickelt sich fort. Offenheit im Zugang zu Bildung, Artikulation, Diskurs und Partizipation könnten ein solches Verständnisses von Bildung in der «nächsten Gesellschaft» begründen.

Waren die allgemeine Schulpflicht und der kostenfreie Zugang zu Bildung wichtige Marksteine, um Bildung zugänglich zu machen, so gilt es zu erarbeiten, wie Bildung als öffentliches Gut im Internet implementiert

3 https://www.saarland.de/it-cluster.htm. 
werden kann: Die Diskussion über «offene» Bildungsressourcen, OnlineKurse und digitale Ökosysteme (Kerres und Heinen 2015) sind Teil des weltweiten Diskurses über «Open Education»:

«Die Digitalisierung eröffnet Freiräume für Bildung: Der Einzelne kann seine Lernprozesse feststellen, visualisieren und mit Anderen reflektieren. Dies kann zu neuen Wegen des Lernens führen, die das Festverdrahtete und Algorithmische übersteigt und Neues für den Einzelnen sichtbar werden lässt.» (Kerres 2018)

Der entscheidende Punkt: Beide Visionen von Bildung sind auf dem Hintergrund der digitalen Technologie denkbar (sie schliessen sich auch nicht grundsätzlich aus) und es ist zu erkennen, wie an verschiedenen Orten beide Visionen Raum greifen. Gesellschaftliche Wirklichkeit entsteht entlang solcher Gestaltungsoptionen und ist als gesellschaftlicher Aushandlungsprozess aufzufassen.

Dabei bestehen nicht für alle Akteure die gleichen Möglichkeiten sich einzubringen; es wäre naiv, diesen Prozess als eine Art demokratische Abstimmung über «die Zukunft» zu verstehen. Vielmehr geschehen diese Prozesse vielfach unterhalb der Wahrnehmungsschwelle, etwa wenn wir Internetseiten aufrufen, uns registrieren und unsere Daten preisgeben, oder wenn sich digitale Unternehmen (ohne Gegenwehr) der Besteuerung in einem Staat entziehen. Die digitale Gesellschaft entsteht in der Schaffung und Nutzung von Handlungsoptionen.

\section{Neue Kompetenzen?}

Im Folgenden wenden wir uns der Frage zu, welche Bildungsinhalte und -ziele «Bildung in der digitalen Welt» ausmachen kann. Auch in dieser Diskussion treffen wir auf eine technikdeterministische Sicht, die davon ausgeht, dass sich die Bildungsinhalte unmittelbar durch die neue Technik ergeben. Die Bedienung des Computers und seiner Anwendungen, mobile Endgeräte und die Gefahren des Internets stellen neue Anforderungen dar. Deswegen, so eine verbreitete Annahme, tritt zu den Grundtechniken des Lesens, Schreibens und Rechnens, als weitere Kulturtechnik, die Computerbeherrschung hinzu: der kompetente Umgang mit digitalen Medien oder kurz «digitale Kompetenz(en)». 
Beispielhaft sei hier auf die Forderung des Aktionsrats Bildung der Vereinigung der bayerischen Wirtschaft (2018) verwiesen:

Bildung in einer digital vernetzten Welt erweitert heute Medienkompetenz um den Begriff der digitalen Souveränität. Neben dem Erlernen der Kulturtechniken Lesen, Schreiben und Rechnen ist der souveräne Umgang mit digitalen Medien die Voraussetzung für eine systematische Verankerung der Medienbildung im Handeln jedes Einzelnen. (ebd., 18)

Bereits 1995 forderte die Bildungskommission NRW zur Zukunft der Schule:

Die Beherrschung moderner Informations- und Kommunikationstechnologien wird zu einer basalen Kulturtechnik werden, deren Stellenwert dem Lesen und Schreiben gleichkommt. ${ }^{4}$

Diese Aussagen versuchen in ihrer programmatischen Anlage ihr Anliegen in der bildungspolitischen Diskussion «nach vorne zu bringen». Die rhetorische Figur dient dazu, die Bedeutung der Thematik herauszustellen sowie Förderprogramme und Entwicklungsmassnahmen zu begründen. Sie hebt das Digitale auf das Niveau von elementaren Grundfertigkeiten und soll die Dringlichkeit von Investitionen verdeutlichen. Es scheint dabei offensichtlich um zusätzliche und neue Kompetenzen zu gehen, vermutlich weil die Technik ja auch als etwas Neues in der Lebens- und Arbeitswelt erlebt wird.

Eine alternative Sicht versteht die Digitalisierung zunächst als etwas, das die Gesellschaft in ihren Funktionssystemen und Handlungspraktiken durchdringt. Im Übergang zur digitalen Epoche entsteht nicht eine weitere, zusätzliche Kulturtechnik, sondern die Digitalisierung durchdringt alle bisherigen Handlungspraktiken, also auch wie wir lesen, schreiben und rechnen. Der epochale Einschnitt wird unterschätzt, wenn das Digitale (nur) als etwas Zusätzliches beschrieben wird, das zu bestehenden Praktiken des gesellschaftlichen Handelns und der Wissenserschliessung

4 Bildungskommission NRW. 1995. Zukunft der Bildung - Schule der Zukunft. Denkschrift der Kommission «Zukunft der Bildung - Schule der Zukunft» beim Ministerpräsidenten des Landes Nordrhein-Westfalen. Neuwied: Luchterhand. 
und -generierung hinzutritt. Während die moderne Gesellschaft sich durch die Rationalität ihrer funktionalen Subsysteme auszeichnet, fungiert die Netzwerkgesellschaft, nach Baecker (2017), als eine offene Ökologie mit einer überraschenden, potenziell flüchtigen Ordnung: «Das irritierbare Netzwerk und die überfordernde Komplexität werden zu Denkfiguren unserer Orientierung.» Es gilt zu verstehen, dass die Digitalisierung diese Praktiken essentiell durchdringt und irritiert.
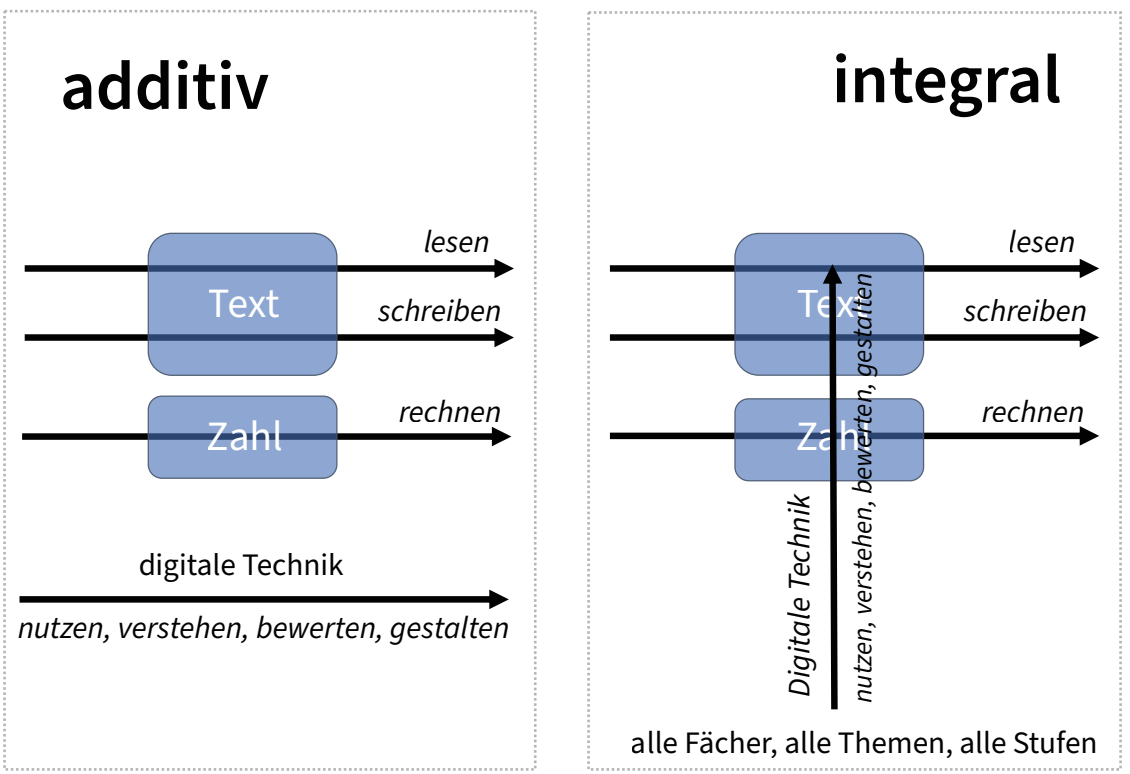

Abb. 2.: Additive vs. integrale Sicht auf Kulturtechniken.

Die binäre Kodierung einer digitalen vs. analogen Welt bleibt dagegen einer Denkfigur verhaftet, die die Reichweite des Digitalen verkennt. Auch die Rede von digitaler Bildung denkt diese in ihrer Differenz zu einer (schwer zu begründenden) «analogen» Bildung. Das Denken entlang einer Grenze des Analogen vs. Digitalen konnotiert das Analoge dabei üblicherweise als etwas Echtes und das Digitale als dazu Defizitäres, etwa wenn es heisst, dass eine «echte Begegnung» nur im «wirklichen Leben» stattfinden kann, wo die Lebenswelt doch längst von der digitalen Technik durchdrungen ist. Dies soll nicht Unterschiede leugnen, die in den verschiedenen Konstellationen menschlicher Begegnungen möglich sind, dennoch regulieren 
sich menschliche Beziehungen längst mit Mitteln des Digitalen, etwa wenn Menschen über social media und Statusmeldungen ihr Leben teilen.

Die Implikationen einer solchen integralen Sicht auf Kompetenzen in der digitalen Epoche sind weitreichend (vgl. Heinen und Kerres 2017). Denn sie sieht die Digitalisierung nicht als zusätzlichen Platz im Gefüge von Curricula, sondern fragt nach den Implikationen für alle vorhandenen Fächer und Stufen, Bildungsinhalte und -institutionen. Dies betrifft immer mehrere Ebenen:

- Wo kommen digitale Werkzeuge ins Spiel, wenn es um die Erschliessung und Erarbeitung von Wissen geht?

- Welche neuen Themen und Inhalte ergeben sich im Zusammenhang mit der Digitalisierung?

- Wie können wir unseren Umgang mit digitaler Technik reflektieren: Welche Implikationen hat die Digitalisierung für mich und unser $\mathrm{Zu}$ sammenleben?

Eine solche Überarbeitung von Lehrinhalten schliesst im Übrigen nicht aus, dass die Thematisierung auch in einem eigenen, ausgewiesenen Zeitfenster im Rahmen schulischer Curricula erfolgt, etwa wenn es um die intensivere Auseinandersetzung mit der Funktionsweise und den Implikationen des Digitalen geht; der Erwerb dieser Kompetenzen lässt sich aber nicht auf ein solches Fach «abschieben», in dem diese Fragen dann isoliert behandelt werden, d.h. ohne dass sich die Arbeit in den anderen Fächern ändern würde. Die interdisziplinäre Diskussion zwischen Informatik und Medienpädagogik im Rahmen der Dagstuhl-Konferenz 2016 hat die Konvergenz der verschiedenen Sichten unterstrichen (vgl. Herzig 2016).

Im Kern lässt sich festhalten, Bildung in der digitalen Epoche geht es weiterhin - um Urteilsfähigkeit im Umgang mit Information und Medien, im Kontext der spezifischen Bedingungen des jeweiligen Mediensystems, das sich in der digitalen Epoche ändert. Auf der einen Seite ist genauer herauszuarbeiten, was die neue Medienumwelt in der digitalen Epoche auszeichnet; auf der anderen Seite wird man im Kern überraschend ähnliche Formulierungen finden, wenn wir - im Folgenden - über Bildungsziele für «Bildung in der digitalen Welt» sprechen. 


\section{Zukunftsentwürfe in Kompetenzkatalogen}

Zur Wende des Jahrhunderts stiess die OECD mit dem Schlagwort $21^{\text {st }}$ century skills eine internationale Diskussion über die Erneuerung von Curricula auf dem Hintergrund der Digitalisierung an. Dede (2010) hat die Diskussion nachgezeichnet, in der vor allem die vier Kompetenzen: Kommunikation, Kollaboration, Kreativität und Kritik, in den Vordergrund gestellt werden, die ein «traditionelles» Lernen mit der Fokussierung auf den Fachunterricht relativieren sollen. Viele gesellschaftliche Gruppen, Verbände, Stiftungen und staatliche Einrichtungen haben entsprechende Positionspapiere zu den neuen Anforderungen in der digitalen Welt vorgelegt und insofern besteht kein Mangel an Referenzen. Neben nationalen, internationalen und supranationalen Einrichtungen (vgl. Binkley u.a. 2012) haben auch Unternehmen und Wirtschaftsverbände (Trilling und Fadel 2009) solche Kataloge vorgelegt, die Kompetenzen benennen, die als für die Zukunftsbewältigung erforderlich benannt werden. Digital literacy kann dort im Kern als die neue Kompetenz identifiziert werden (Mishra, Koehler, und Henriksen 2011) und steht im Mittelpunkt vieler Kompetenzkataloge (s.a. Ilomäki u.a. 2016).

Wie lassen sich diese Papiere in die bildungswissenschaftliche Diskussion grundsätzlich einordnen? Aussagen über Technikzukünfte und ihre Kompetenzanforderungen können als Versuche verstanden werden, Kontingenz im Umgang mit einer - durch die Technikentwicklung verunsicherten - Zukunft zu reduzieren. In der Analyse der Kataloge lassen sich mögliche Rückwirkungen der zumeist impliziten Zukunftsentwürfe für den gesellschaftlichen Diskurs untersuchen (Grunwald 2012; Häußling 2014). Dabei wird deutlich, dass diese Konzepte davon ausgehen, a) Zukunft vorhersagen zu können, und b) dass die Zukunft durch die Technik determiniert ist: Wenn wir die Technik von Morgen kennen, wissen wir wie Zukunft aussieht, weil Technik die Zukunft erzeugt.

Der Ausweisung von Kompetenzkatalogen liegt immer eine Annahme über Zukünfte zugrunde. Wenn formuliert wird, wir brauchen Neugier, Mut und Selbstständigkeit verbindet sich damit eine andere Projektion als die Forderung von Empathie, Teamfähigkeit oder Verantwortung. Gleichzeitig besteht die Besonderheit dieser Diskussion darin, dass genau diese hinterlegten Zukunftsentwürfe regelmässig «versteckt» werden und 
damit einer Auseinandersetzung entzogen werden. Die entsprechenden Kompetenzkataloge basieren auf Annahmen, wie Technik, Arbeit, Berufe und Gesellschaft künftig sein werden. Die künftigen Anforderungen an Bildungsarbeit werden durch Rückgriff auf eine angenommene Zukunft formuliert. Die aus solchen Zukunftsentwürfen «abgeleiteten» Kompetenzkataloge verraten allerdings nicht mehr, auf Grund welcher Zukunftserwartung diese oder jene Kompetenz begründet wird. Sie werden versteckt und entziehen sich damit dem gesellschaftlichen Diskurs. Dabei bleibt auch brisant, wie solche Papiere entstehen. Voogt et al. (2013) rekonstruieren, wie diese Papiere in politischen Abstimmungsprozessen entstehen und die Frage bleibt, welche Personen und Institutionen hierbei besonderes Gewicht erhalten (s.a. Rotherham und Willingham 2010).

In der deutschsprachigen Diskussion haben die Papiere $\mathrm{zu}_{21} 1^{\text {st }}$ century skills und zu weiteren Schlagworten, wie das OECD-Learning Framework $2030^{5}$ oder die Kompetenzkataloge der Europäischen Kommission zu DigComp $^{6}$, im Vergleich zu anderen Ländern auffallend wenig Aufmerksamkeit erlangt; vielleicht weil der Bildungsbegriff immer schon eine breitere Anlage von Curricula und Bildungszielen impliziert (s. aber Petko, Döbeli Honegger, und Prasse 2018) und das Konstrukt einer Allgemeinbildung Grundlage für die weitere Diskussion darstellen kann.

\section{Medienkompetenz und Bildung}

Im deutschsprachigen Raum steht dagegen seit den 1970er Jahren der Begriff der Medienkompetenz im Mittelpunkt der Diskussion. Wenn die Schule auf das Buch fixiert war, wuchs die Bedeutung von Radio, Fernsehen, Kino und Zeitschriften in der Freizeit. «Medienkompetenz» nahm diese «neuen» Medien in den Blick und zielt auf den kritischen Umgang mit diesen (Moser, Grell, und Niesyto 2011; Groeben und Hurrelmann 2002). Anders als von Baacke (1973) ursprünglich intendiert, wird dabei Medienkompetenz oft auf die Fertigkeiten im Umgang mit analogen oder digitalen Medien reduziert. Aufenanger forderte bereits (2000):

5 http://www.oecd.org/education/2030/learning-framework-2030.htm.

6 https://ec.europa.eu/jrc/en/digcomp/digital-competence-framework. 
«Diese sozialtechnologische und affirmative Variante des Begriffs der Medienkompetenz, die dieser auch sehr schnell nahe legt, muss überwunden und durch die grundlegenden Aspekte von Erziehung und Bildung ergänzt werden.»

Mit Medienkompetenz verbinden sich in der öffentlichen Auffassung oft Trainings zur Computerbedienung oder auch Veranstaltungen, in denen vor den Gefahren der digitalen Medien gewarnt wird und über die Handynutzung an Schulen oder Cybermobbing gesprochen wird. In den 1990er Jahren rückt deswegen die Beschäftigung mit dem Begriff der Medienbildung in den Vordergrund. Dabei wird auf das weitere Verständnis eines Bildungsbegriffs verwiesen und eine Alternative für die in der Öffentlichkeit verbreitete Reduktion von Medienkompetenz auf Bedienfertigkeiten angeboten (Aufenanger 1999; Marotzki und Jörissen 2008; Tulodziecki 2015).

Der 2010 vorgelegte Bericht der vom BMBF beauftragten Expertenkommission markiert einen Einschnitt der Diskussion. Er erschien unter dem Titel: «Kompetenzen in einer digital geprägten Kultur» (Expertenkommission des BMBF zur Medienbildung 2010). Damit wurde signalisiert, dass es nicht mehr um Medienkompetenz als abgesetztem Kompetenzbereich geht, sondern eben um Kompetenzen für eine «digital geprägte Kultur» geht. Benannt wurden:

a. Information und Wissen: Zugang zum Wissen einer Kultur,

b. Kommunikation und Kooperation: Teilhabe am gesellschaftlichen Diskurs,

c. Identitätssuche und Orientierung: Entwicklung von Persönlichkeit und

d. digitale Wirklichkeiten und produktives Handeln: (selbständige) Aneignung und Nutzung von digitaler Technik mit Bezug zur Lebens- und Arbeitswelt.

Diese Argumentation schliesst an einen Bildungsbegriff an, der Bildung als ein reflektiertes Verhältnis des Menschen zu den Dingen, zu den Anderen und zu sich versteht: als ein Sich-ins-Verhältnis-Setzen zur Welt (etwa bei Marotzki 1990; Meder 2007). Bildung in der digitalen Welt oder Bildung in einer digital geprägten Kultur umgeht zunächst die sprachlichen 
Schwierigkeiten, die mit der Formulierung einer digitalen Bildung verbunden sind. Vor allem aber zeigen sie an: Es geht im Kern um Bildung, und diese gilt es in Relation zu den Veränderungen zu befragen, die wir im Zusammenhang mit der digitalen Technik erfahren. Dies ist ein wesentlicher Perspektivwechsel, auch zu dem Konstrukt Medienkompetenz, wenn es als additiv zu anderen Kompetenzen betrachtet wird.

Die Kultusminister der Bundesländer in der Bundesrepublik Deutschland veröffentlichten im Dezember 2016 das Strategiepapier zu «Bildung in einer digitalen Welt» ${ }^{7}$, welches diesen Überlegungen Nachdruck verleiht, und eine entsprechende Überarbeitung der schulischen Bildungsstandards eingeleitet hat:

«Wenn der schulische Bildungsauftrag sich in der ,digitalen Welt' nachhaltig verändert, dann wird perspektivisch Medienbildung keine schulische Querschnittsaufgabe mehr sein, sondern integraler Bestandteil aller Unterrichtsfächer.»

Damit wird deutlich: Wenn die Digitalisierung die Lebens-, Lern- und Arbeitswelt durchdringt, erfahren wir diese wesentlich durch digitale $\mathrm{Me}$ dien vermittelt. Unsere Teilhabe an Kultur, die Kommunikation mit Anderen basiert auf digitalen Medien und auch die Sicht auf uns selbst wird beeinflusst durch Artefakte, die wir mit digitalen Werkzeugen erzeugen. Lernen geht dabei mit dem Kompetenzerwerb einher, mit dem ich etwas Bestimmtes beherrsche. Wie dies im Fachunterricht umgesetzt werden kann, ist Thema der Fachdidaktik.

Grundsätzlicher verweist eine strukturale Bildungstheorie auf die Bedeutung von Unbestimmtheitsräumen des Lernens (Jörissen und Marotzki 2009, 102), in denen sich das Verhältnis des Menschen zu sich oder zur Welt ändern kann: «Bildungsprozesse werden dort als höherstufige Lernprozesse verstanden, bei denen nicht nur neues Wissen angeeignet wird, sondern auch das Welt- und Selbstverhältnis des Subjekts eine grundlegende Transformation erfährt» (Koller 2016, 149). Einem solchen Bildungsverständnis «geht es weniger um die Inhalte der jeweiligen Medien, sondern um ihre strukturalen Aspekte» (Marotzki und Jörissen 2008, 103) des Welt- und

7 https://www.kmk.org/fileadmin/Dateien/pdf/PresseUndAktuelles/2016/Bildung_digitale_Welt_Webversion.pdf. 
Selbstverhältnisses. In den Fokus rückt dann, was die pragmatistischen Bildungstheorie als bildende Erfahrung bezeichnet und die Frage, wie Erfahrungen möglich werden, die irritieren und Bedeutung entwickeln (vgl. Kerres und de Witt, 2004).

Dieses Verständnis von Bildung passt zu den kulturtheoretisch begründeten Merkmalen des Digitalen: Wenn sich die digitale Epoche durch Vernetztheit, Ambivalenz und Unbestimmtheit auszeichnet (Stalder 2016) dann sollte eine «bildende» Qualität von Lernen für die Bewältigung dieser Herausforderung geeignet sein. Das Verständnis von Bildung bedarf damit im Kontext der Digitalisierungsdiskussion keiner grundsätzlichen Erneuerung. Freilich wären im nächsten Schritt die Kompetenzen zu betrachten, die Voraussetzung für «Bildung in der digitalen Welt» sind.

\section{Kompetenzen für «Bildung in der digitalen Welt»}

Bildung (in der digitalen Welt) ist mehr als die Summe erworbener Kompetenzen im Umgang mit einer durch digitale Medien geprägten Kultur; doch gleichzeitig bedarf sie verschiedener Kompetenzen, um die Gestaltungsoptionen einer digitalen Welt nutzen zu können. Gehen wir zurück auf die ursprünglichen Kataloge von Baacke (1973) und Groeben und Hurrelmann (2002), wie sie im Kontext der elektronischen Massenmedien entwickelt worden sind. Beziehen wir diese auf die Digitalisierung, so lassen sich die sieben Kompetenzbereiche für Bildung in der digitalen Welt in Abbildung 3 reformulieren, die sich auf Wissen, Fertigkeiten und Einstellungen beziehen. Dabei geht es um Anliegen nicht nur der Medienpädagogik, sondern auch benachbarter Disziplinen innerhalb und ausserhalb der Erziehungswissenschaft:

1. Wissen über Medien (u.a. in der Medienpädagogik, Informatik- und Deutschdidaktik oder den Informationswissenschaften thematisiert),

2. Funktionen verstehen (insbesondere in der Informatik- und Technikdidaktik aufgegriffen),

3. Medien nutzen (ebenfalls Thema etwa der Informatik- und Technikdidaktik),

4. mit Informationen umgehen (in der Informationswissenschaft als Informationskompetenz diskutiert), 
5. Medien gestalten (traditioneller Fokus handlungsorientierter Ansätze der Medienpädagogik),

6. eigenes Medienverhalten reflektieren (insbesondere in Bildungstheorien diskutiert),

7. gesellschaftliche Implikationen bewerten (immer schon Anliegen von Medienkritik und sozialwissenschaftlicher Diskurse).

7. gesellschaftliche Implikationen bewerten

- Was bedeutet Digitalisierung für die Gesellschaft?

- Wo entsteht Ungleichheit, Monopolisierung oder Ausgrenzung?

- Wie kann ich mich einbringen in den Diskurs?

6. das eigene Medienverhalten reflektieren

- Wie nutze ich Medien?

- Wie stelle ich mich in Medien dar?

5. Medien gestalten

- Wie produziere ich Medien?

- Wie kann ich Medien gestalten?

4. mit Informationen umgehen

- Wie finde ich Informationen?

- Wie bewerte ich Informationen?

- Wie gehe ich mit Informationen um?

\section{Medien nutzen}

- Wie bedient man digitale Medientechnik?

- Wie werden Computeranwendungen genutzt?

2. Funktionen verstehen

- Wie funktioniert digitale Technik und das Internet?

- Was sind Algorithmen, Daten, Speicher etc.?

1. Medien kennen

- Was gibt es für Plattformen, Angebote, Werkzeuge?

- Was kann man damit machen?

- Was sind die Probleme?

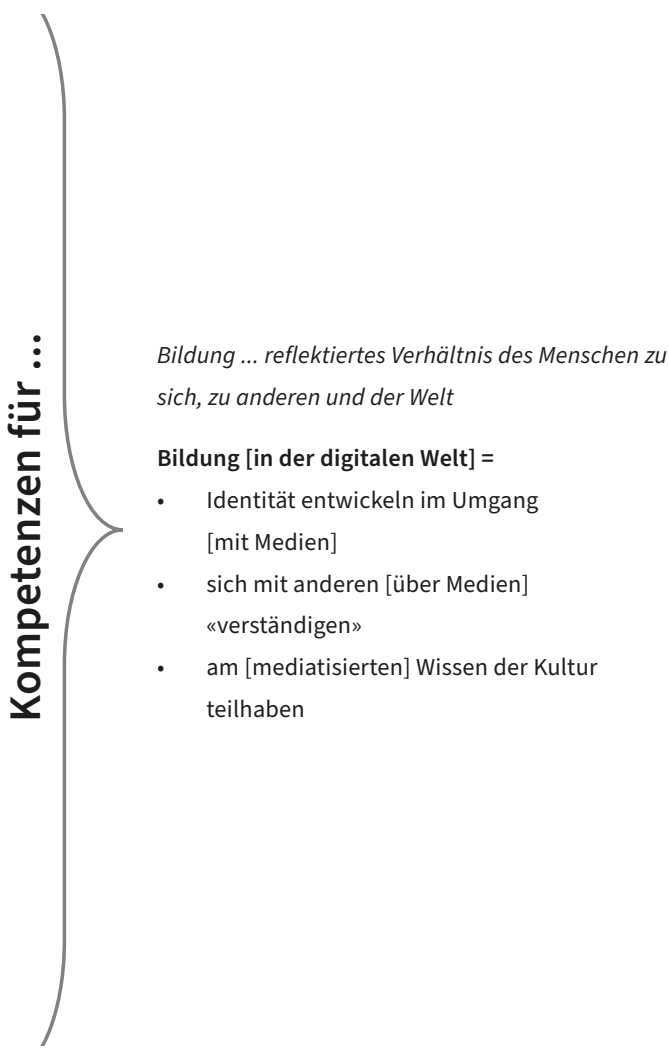

Abb. 3.: Kompetenzen für Bildung [in der digitalen Welt].

Die genannten Kompetenzen umfassen Wissen, Fertigkeiten und Einstellungen und beziehen sich gleichermassen auf Sach-, Selbst- und Sozialkompetenz. Entlang dieser Kompetenzbereiche lässt sich auch näher untersuchen, wo sich tatsächlich «neue» Kompetenzen herauskristallisieren. Die Vermutung wäre, dass sich eher in den unteren Kompetenzbereichen 
(Medien kennen, verstehen, nutzen) «neue», die digitale Epoche prägende Kompetenzen finden, während bei den höheren Kompetenzbereichen (mit Information umgehen, Medien gestalten etc.) auf Kompetenzen zurückgegriffen werden kann, die bereits im Umgang mit früheren Medientechniken angelegt sind.

In der Auseinandersetzung mit diesen Kompetenzen muss sich die Medienpädagogik mit den benachbarten Disziplinen beschäftigen, die ihrerseits manchmal eher abgeschottet sich mit den Kompetenzen im Kontext der Digitalisierung beschäftigen. So lässt sich Medienkompetenz, wie in der Medienpädagogik diskutiert, und Informationskompetenz, wie in der Informationswissenschaft behandelt, kaum systematisch trennen. Letztlich fliessen sie ein in «die Kompetenzen» für Bildung in der digitalen Welt.

In der Darstellung in Abb. 3 soll deutlich werden, dass und wie sich ein Bildungs- und Kompetenzbegriff ergänzen, wenn es um die digitale Epoche geht. Pietraß (2011) hatte bereits aufgezeigt, dass und wie sich der Bildungs- und Kompetenzbegriff in der medienpädagogischen Diskussion ergänzen können (ebd., 132):

Der Kompetenzbegriff ist gebunden an einen technisch-sozialen Zeithorizont und bezieht daraus seine für die Medienpädagogik unverzichtbare gesellschaftspolitische Bedeutung, während der Bildungsbegriff seine Verbindlichkeit aus einer jahrhundertealten Vorstellung von reflektierender Individualität und im Handeln verantworteter Sozialität bezieht, welche anthropologisch begründet wird - obwohl ihm dort, wo Bildungsdimensionen mit Könnensforderungen ausformuliert werden, eine ähnliche Aufgabe wie dem Kompetenzbegriff übergeben und er ähnlich zeitbezogen aktualisiert wird.

Den Bildungsbegriff betrachten wir hier vor allem als Zielhorizont. Er entwirft die Perspektive, auf die die zu erwerbenden Fähigkeiten bezogen werden können, und kann an bildungstheoretische Ansätze anschliessen die Bildung als reflektiertes Weltverhältnis beschreiben, in der die Person am (mediatisierten) Wissen der Kultur teilhat, in der sie die eigene Identität im Umgang mit Medien entwickelt und sich mit anderen über Medien «verständigt». Zugleich trägt die Beschäftigung mit den Kompetenzen zur Konkretisierung bei und hilft bei der Ausarbeitung von Curricula: Welche 
Voraussetzungen sind aus Sicht der verschiedenen Disziplinen nötig, um die angestrebten Zielformulierungen einzulösen?

In der neu entstehenden Diskussion über post-digitale Bildung und Kultur wird Digitalisierung als Transition aufgefasst. Von der Perspektive einer Welt ausgehend, in der das Digitale bereits «verarbeitet» ist, blickt sie auf jüngste Diskurse, als Ausgangspunkt für eine Rekonstruktion der Jetztzeit, um deren Sinnstrukturen zu heben (vgl. Jandrić u.a. 2018 im Editorial für die neue Zeitschrift «Postdigital Science and Education»). Sie wendet den Blick weg von der Digitalisierung, um die Digitalisierung zu verstehen (vgl. die Analyse von Lernpraktiken bei Ryberg, Davidsen, und Hodgson 2018). Eine Methode besteht darin, die Figur des «Digitalen» als gegeben anzunehmen und aus aktuellen Argumentationen herauszunehmen (s. die Ausklammerungen in Abbildung 3). Dabei wird die Kontinuität erkennbar, die den erforderlichen Kompetenzen im Umgang mit lebensweltlichen Anforderungen, Informationen und Medien zugrunde liegen. Gleichzeitig ist die neue Gemengelage der «nächsten Gesellschaft» einzuarbeiten, die nicht mehr auf die «digitale Souveränität» des einzelnen Individuums setzt, sondern mit einem Kontrollüberschuss und mit der Unberechenbarkeit des Berechneten rechnet.

Die Kompetenzen für Bildung in der digitalen Welt entwickeln sich in der handelnden Auseinandersetzung des Menschen mit seiner Umwelt. Es bleibt die Frage für die weitere Forschung, inwieweit die in Abbildung 3 skizzierten sieben Kompetenzen an bestimmte Domänen (wie z.B. Sprachen oder Naturwissenschaften) gebunden sind oder sich als generisch erweisen (also nicht an Domänen gebunden sind). Dies ist von Bedeutung, da Bildungsangebote anders anzulegen sind, wenn Kompetenzen entweder unabhängig oder nur innerhalb von Domänen erworben werden. Wir können davon ausgehen, dass es Kompetenzen gibt, die auf neue Anforderungen transferiert werden können, und andere Kompetenzen, die stärker an Situationen, Medientechniken und thematische Kontexte gebunden sind. Weniger kontextgebunden wäre die Fertigkeit, Information im Netz zu suchen, oder das Wissen um Kriterien zur Bewertung der Qualität von Informationen. Die Fertigkeit, eine Statistiksoftware kompetent zu nutzen, bezieht sich dagegen auf einen engeren Kontext, und sie ist wenig übertragbar auf andere Kontexte. 
Dabei lässt sich momentan nicht bestimmen, ob und an welchen Stellen «generische» Kompetenzen für Bildung in der digitalen Welt entstehen, die transferierbar sind, und wo wir von stärker domänengebundenen Kompetenzen ausgehen müssen, die sich auf eine spezifische Technik in einer (Klasse von) Anwendungssituation bezieht. Die Lehr-Lernforschung zeigt, dass viele Kompetenzen stärker domänengebunden und damit weniger leicht transferierbar sind als vielfach angenommen wird (Mähler und Stern 2006; Prenzel 2010). Die medienpädagogische Diskussion geht in der Regel davon aus, dass «Medienkompetenz» generisch angelegt ist, d.h. eine Person wird in der Auseinandersetzung mit verschiedenen Medientechniken (Buch, Fernsehen, Internet), in verschiedenen Kontexten (Schule, Freizeit, Beruf) und Inhalten (politische Nachrichten, Spielfilm, Twitternachricht) entweder eine hohe oder eine niedrige Medienkompetenz aufweisen - und zwar durchgängig. Betrachten wir die Ubiquität des Medialen in der digitalen Epoche wäre der Frage nachzugehen, inwieweit diese Annahme zutrifft. Auch hier könnte man (auch mit Bloom) annehmen, dass die «niedrigeren» Kompetenzen in Abb. 3 eher domänenabhängig sind, während die «höheren» Kompetenzen eher domänenunabhängig sind.

\section{Schluss}

Die Unsicherheit anerkennend, die in jeder Selbstbeobachtung besteht, spricht Baecker (2007; 2018) von der «nächsten Gesellschaft», die er als epochalen Einschnitt im Übergang von einer durch den Buchdruck geprägten Gesellschaft der Moderne zu einer vernetzten Gesellschaft beschreibt (s.a. Castells 2004). In diesem, bislang eher schemenhaft zu erkennenden Übergang sind andere Lösungen zu finden für die Fragen, die vorige Gesellschaften bereits beantwortet haben. Die Rhetorik der sich anbahnenden Bildungsrevolutionen ebenso wie kulturpessimistische Zeitdiagnosen eines kulturellen Zerfalls sind Indizien für den gesellschaftlichen Suchprozess, in dem neue Ordnungen und Semantiken als Antwort auf den Sinnüberschuss des neuen Mediums zu finden sind (s.a. Allert und Richter 2017; Wunder 2018). In der Befürwortung wie der Kritik ist dabei die Gewissheit einer Zeitdiagnose hinterlegt, die angesichts der Ambivalenzen, mit denen wir konfrontiert sind, schwierig aufrecht zu erhalten ist. 
In der Rede von den Wirkungen des Digitalen kommt für den gesellschaftlichen Diskurs hinzu, dass sie ausgesprochen «wirksam» ist, allerdings anders als die Kontrahenten es vermuten. Gegner wie Befürworter schreiben der Technik die Fähigkeit zu, eine bestimmte Zukunft zu erzeugen (und in dem Medium der Artikulation dieser Zuschreibung tragen sie zur Erzeugung dieser Zukunft bei.) In ihren zugrundeliegenden Annahmen über die Wirkung von Technik gleichen sie sich: Der Mensch ist der Technik ausgeliefert, er kann versuchen, die Entwicklung zu beschleunigen oder zu verhindern. Vielleicht in Voraussicht auf die kommende «künstliche Intelligenz», wird die Entwicklung als «unausweichlich» kommuniziert und die Gestaltungsoptionen der digitalen Epoche bleiben verdeckt. Im Kern geht es deswegen darum, die digitale Epoche als einen gesellschaftlichen Aushandlungsprozess zu verstehen, der sich gegen die Rede von den Wirkungen des Digitalen stellt.

Das Digitale ist dabei nicht additiv in der Bildungsarbeit zu verstehen, sondern es durchdringt unsere Lebens- wie auch Lernwelten. Neue, sogenannte digitale Kompetenzen sind dabei auffallend schwer zu begründen. Bei genauerer Betrachtung finden sich grundlegende Anforderungen, die mit einem Verweis auf den Bildungsbegriff und die Idee einer allgemeinen Bildung ebenso wie die grundlegenden Kompetenzen im Umgang mit Informationen und Medien gut beantwortet werden können. Bildungs- und Kompetenzbegriff sind häufig als kontrovers und als Alternativen diskutiert worden, wohingegen Pietraß (2011) ihr Zusammenwirken als Chance skizziert. Der Argumentation folgend wären Bildungs- und Kompetenzbegriff als Relation weiter zu entwickeln: «Bildung (in der digitalen Welt)» verweist dabei auf Zielhorizonte, für die sieben Kompetenzen für die gestaltende Teilhabe benannt wurden. In der weiteren Ausarbeitung der Kompetenzen wäre zu prüfen, ob und inwiefern die beschriebenen Kompetenzen die Sichten verschiedener Disziplinen hinreichend inkludieren, etwa der Informations- und Bibliothekswissenschaft, der Informatik- und Technikdidaktik oder weiterer Fachdidaktiken. Es bleibt im weiteren genauer auszuloten, an welchen Stellen wir das Digitale unterschätzen, etwa wenn wir die Idee einer Souveränität des Internet-Nutzenden aufrecht erhalten wollen, und wo wir uns von der Macht des Digitalen täuschen 
lassen, die uns Neues verspricht, wo doch die Besinnung und das Beharren auf Altbekanntes die Antwort sein kann.

\section{Literatur}

Ackeren, Isabell van, Albert Bilo, Uwe Blotevogel, Holger Gollan, Sandrina Heinrich, Patrick Hintze, Julia Liebscher, und Anke Petschenka. 2017. «Vom Strategiekonzept zur Entwicklung der Lehr-/Lernkultur? Ein Überblick über bisherige Rahmenbedingungen und Maßnahmen der E-Learning Strategie». In Flexibles Lernen mit digitalen Medien ermöglichen - Strategische Verankerung und Erprobungsfelder guter Praxis an der Universität Duisburg-Essen, herausgegeben von Isabell van Ackeren, Michael Kerres, und Sandrina Heinrichs, 35-55. Münster: Waxmann.

Allert, Heidrun, Michael Asmussen, und Christoph Richter. 2018. «Formen von Subjektivierung und Unbestimmtheit im Umgang mit datengetriebenen Lerntechnologien - eine praxistheoretische Position». Zeitschrift für Erziehungswissenschaft 21 (1): 142-58. https://doi.org/10.1007/s11618-017-0778-7.

Allert, Heidrun, und Christoph Richter. 2017. «Kultur der Digitalität statt digitaler Bildungsrevolution». Pädagogische Rundschau 71 (1): 19-32.

Aufenanger, Stefan. 1999. «Medienkompetenz oder Medienbildung? Wie die neuen Medien Erziehung und Bildung verändern». Bertelsmann Briefe, Nr. 142: 21-24.

Aufenanger, Stefan. 2000. «Medien-Visionen und die Zukunft der Medienpädagogik. Plädoyer für Medienbildung in der Wissensgesellschaft.» medien praktisch. Zeitschrift für Medienpädagogik, Nr. 93: 4-8.

Baacke, Dieter. 1973. Kommunikation und Kompetenz. Grundlegung einer Didaktik der Kommunikation und ihrer Medien. München.

Baecker, Dirk. 2007. Studien zur nächsten Gesellschaft. Frankfurt: Suhrkamp.

Baecker, Dirk. 2017. «Wie verändert die Digitalisierung unser Denken und unseren Umgang mit der Welt?» In Handel 4.0: Die Digitalisierung des Handels Strategien, Technologien, Transformation, herausgegeben von Rainer Gläß und Bernd Leukert, 3-24. Berlin, Heidelberg: Springer. https://doi.org/10.1007/9783-662-53332-1_1.

Baecker, Dirk. 2018. 4.0 oder Die Lücke die der Rechner lässt. Berlin: Merve.

Bernard, Robert M., Eugene Borokhovski, Richard F. Schmid, und Rana M. Tamim. 2018. "Gauging the Effectiveness of Educational Technology Integration in Education: What the Best-Quality Meta-Analyses Tell Us». In Learning, Design, and Technology: An International Compendium of TheorY, Research, Practice, and Policy, herausgegeben von Michael J Spector, Barbara B Lockee, und Marcus D. Childress, 1-25. Cham: Springer International Publishing. https:// doi.org/10.1007/978-3-319-17727-4_109-2. 
Bernard, Robert M., Eugene Borokhovski, Richard F. Schmid, Rana M. Tamim, und Philip C. Abrami. 2014. "A Meta-Analysis of Blended Learning and Technology Use in Higher Education: From the General to the Applied». Journal of Computing in Higher Education 26 (1): 87-122. https://doi.org/10.1007/s12528013-9077-3.

Binkley, Marilyn, Ola Erstad, Joan Herman, Senta Raizen, Martin Ripley, May Miller-Ricci, und Mike Rumble. 2012. "Defining Twenty-First Century Skills». In Assessment and Teaching of 21st Century Skills, herausgegeben von Patrick Griffin, Barry McGaw, und Esther Care, 17-66. Dordrecht: Springer Netherlands. https://doi.org/10.1007/978-94-007-2324-5_2.

Castells, Manuel. 2004. Die Internet-Galaxie: Internet, Wirtschaft und Gesellschaft. Wiesbaden: Verlag für Sozialwissenschaften.

Clark, Richard E. 1983. "Reconsidering Research on Learning from Media». Review of Educational Research 53 (4): 445-59. https://doi. org/10.3102/00346543053004445.

Clark, Richard E. 1994. "Media Will Never Influence Learning». Educational Technology Research and Development 42 (2): 21-29. https://doi.org/10.1007/ BFo2299088.

Dede, Chris. 2010. "Comparing Frameworks for 21st Century Skills». In 21st Century Skills: Rethinking How Students Learn, herausgegeben von James A. Bellanca und Ron Brandt, o.S. Solution Tree Press.

Dräger, Jörg, und Ralph Müller-Eiselt. 2015. Die digitale Bildungsrevolution: Der radikale Wandel des Lernens und wie wir ihn gestalten können. München: Deutsche Verlags-Anstalt.

Expertenkommission des BMBF zur Medienbildung. 2010. «Kompetenzen in einer digital geprägten Kultur. Medienbildung für die Persönlichkeitsentwicklung, für die gesellschaftliche Teilhabe und für die Entwicklung von Ausbildungsund Erwerbsfähigkeit.» Herausgegeben von Bundesministerium für Bildung und Forschung (BMBF). http://www.dlr.de/pt/Portaldata/45/Resources/a_dokumente/bildungsforschung/Medienbildung_Broschuere_2010.pdf.

Ferguson, Rebecca, Andrew Brasher, Doug Clow, Dai Griffiths, und Hendrik Drachsler. 2016. «Learning Analytics: Visions of the Future». Edinburgh, Scotland. http://oro.open.ac.uk/45312/.

Groeben, Norbert, und Bettina Hurrelmann. 2002. Medienkompetenz. Voraussetzungen, Dimensionen, Funktionen. Weinheim: Juventa.

Grunwald, Armin. 2012. Technikzukünfte als Medium von Zukunftsdebatten und Technikgestaltung. Bd. 6. Karlsruher Studien Technik und Kultur. Karlsruhe: KIT Scientific Publishing.

Häußling, Roger. 2014. Techniksoziologie. UTB.

Heimann, Paul. 1976. Didaktik als Unterrichtswissenschaft. Stuttgart: Klett. 
Heinen, Richard, und M. Kerres. 2017. " "Bildung in der digitalen Welt" als Herausforderung für Schule». DDS - Zeitschrift für Erziehungswissenschaft, Bildungspolitik und pädagogische Praxis 109 (2).

Herzig, Bardo. 2016. «Medienbildung und Informatische Bildung - Interdisziplinäre Spurensuche». MedienPädagogik: Zeitschrift für Theorie und Praxis der Medienbildung 25 (Computer Science Education), 59-79. https://doi.org/10.21240/ mpaed/25/2016.10.28.X.

Heyer, Paul. 2003. Harold Innis. Rowman \& Littlefield.

Ilomäki, Liisa, Sami Paavola, Minna Lakkala, und Anna Kantosalo. 2016. «Digital Competence - an Emergent Boundary Concept for Policy and Educational Research». Education and Information Technologies 21 (3): 655-79. https://doi. org/10.1007/s10639-014-9346-4.

Jäckel, Michael. 2010. «Mediensoziologie». In Handbuch Spezielle Soziologien, herausgegeben von Georg Kneer und Markus Schroer, 277-94. Wiesbaden: VS Verlag für Sozialwissenschaften. https://doi.org/10.1007/978-3-531-920276_15.

Jandrić, Petar, Jeremy Knox, Tina Besley, Thomas Ryberg, Juha Suoranta, und Sarah Hayes. 2018. "Postdigital science and education». Educational Philosophy and Theory 50 (10): 893-99. https://doi.org/10.1080/00131857.2018.1454000.

Jörissen, Benjamin, und Winfried Marotzki. 2009. Medienbildung- eine Einführung. Stuttgart: UTB.

Kerres, Michael. 2003. «Zu Wirkungen und Risiken neuer Medien in der Bildung: Warum Medien keine Arznei für die Bildung sind.» In Aktuelles und Querliegendes zur Didaktik und Curriculumentwicklung, 261-78. Bielefeld: Janus Presse.

Kerres, Michael. 2017. «Lernprogramm, Lernraum oder Ökosystem? Metaphern in der Mediendidaktik». In Jahrbuch Medienpädagogik 13, herausgegeben von Kerstin Mayrberger, Johannes Fromme, und Petra Grell, 15-28. Berlin: Springer.

Kerres, Michael. 2018. Mediendidaktik. Konzeption und Entwicklung digitaler Lernangebote. 5. Aufl. Berlin: de Gruyter Oldenbourg.

Kerres, Michael, und Claudia de Witt. 2004. «Pragmatismus als theoretische Grundlage zur Konzeption von eLearning». In Handlungsorientiertes Lernen und eLearning. Grundlagen und Beispiele, herausgegeben von D. Treichel und H.O. Meyer. München: Oldenbourg.

Kerres, Michael, und Richard Heinen. 2015. "Open Informational Ecosystems: The missing link for sharing resources for education». International Review of Research in Open and Distance Learning 16 (1).

Köchling, Alina, und Shirin Riazy. 2019. «Fluch oder Segen?. Big Data und Learning Analytics im Lernkontext». weiter bilden. DIE Zeitschrift für Erwachsenenbildung, Nr. 4: 17-20. 
Koller, Hans-Christoph. 2016. "Ist jede Transformation als Bildungsprozess zu begreifen?» In Von der Bildung zur Medienbildung, herausgegeben von Dan Verständig, Jens Holze, und Ralf Biermann, 149-61. Medienbildung und Gesellschaft. Wiesbaden: Springer Fachmedien Wiesbaden. https://doi. org/10.1007/978-3-658-10007-0_8.

Kozma, R.B. 1991. «Learning with media». Review of educational research 61MKCBT5: 179-211.

Krommer, Axel, Martin Lindner, Philippe Wampfler, Dejan Mihajlović, und Jöran Muuß-Merholz. 2019. Routenplaner \#digitale Bildung: Auf dem Weg zu zeitgemäßer Bildung. Eine Orientierungshilfe im digitalen Wandel. 2. Aufl. Booklink.

Kulik, J.A., C.C. Kulik, und P.A. Cohen. 1980. «Effectiveness of computer based college teaching: A meta-analysis of findings». Review of educational research 50: $524-44$.

Latour, Bruno. 1996. «On actor-network theory: A few clarifications». Soziale Welt 47 (4): 369-81.

Lin, Carolyn A. 1996. «Looking back: The contribution of Blumler and Katz's uses of mass communication to communication research». Journal of Broadcasting \& Electronic Media 40 (4): 574-81. https://doi.org/10.1080/08838159609364379.

Mähler, Claudia, und Elsbeth Stern. 2006. «Transfer». In Handwörterbuch Pädagogische Psychologie, herausgegeben von Dieter Rost, 3. Aufl., 782-93. Weinheim: Beltz.

Marotzki, Winfried. 1990. Entwurf einer strukturalen Bildungstheorie: biographietheoretische Auslegung von Bildungsprozessen in hochkomplexen Gesellschaften. Weinheim: Deutscher Studien Verlag.

Marotzki, Winfried, und Benjamin Jörissen. 2008. «Medienbildung». In Handbuch Medienpädagogik, herausgegeben von Uwe Sander, Friederike von Gross, und Kai-Uwe Hugger, 100-109. Wiesbaden: VS Verlag für Sozialwissenschaften. https://doi.org/10.1007/978-3-531-91158-8_11.

Meder, Norbert. 2007. «Theorie der Medienbildung. Selbstverständnis und Standortbestimmung der Medienpädagogik». In Jahrbuch Medienpädagogik 6, herausgegeben von Werner Sesink, M Kerres, und H Moser, 55-73. Wiesbaden: VS Verlag.

Mishra, Punya, Matthew J. Koehler, und Danah Henriksen. 2011. «The seven trans-disciplinary habits of mind: Extending the TPACK framework towards 21st century learning». Educational TechnologY, 22-28.

Moser, Heinz, Petra Grell, und Horst Niesyto, Hrsg. 2011. Medienbildung und Medienkompetenz: Beiträge zu Schlüsselbegriffen der Medienpädagogik. München: Kopäd.

Nassehi, Armin. 2019. Muster: Theorie der digitalen Gesellschaft. 2. Aufl. München: C.H.Beck.

O'Neil, Cathy. 2016. Weapons of Math Destruction: How Big Data Increases Inequality and Threatens Democracy. New York: Crown. 
Petko, Dominik, Beat Döbeli Honegger, und Doreen Prasse. 2018. «Digitale Transformation in Bildung und Schule: Facetten, Entwicklungslinien und Herausforderungen für die Lehrerinnen-und Lehrerbildung». Beiträge zur Lehrerinnen-und Lehrerbildung 36 (2): 157-74.

Pietraß, Manuela. 2011. «Medienkompetenz oder Medienbildung? Zwei unterschiedliche theoretische Positionen und ihre Deutungskraft». MedienPädagogik: Zeitschrift für Theorie und Praxis der Medienbildung 20 (Medienbildung - Medienkompetenz), 121-35. https://doi.org/10.21240/mpaed/20/2011.09.16.X.

Prenzel, Manfred. 2010. «Geheimnisvoller Transfer?» Zeitschrift für Erziehungswissenschaft 13 (1): 21-37.

Ramge, Thomas, und Viktor Mayer-Schönberger. 2017. Das Digital: Markt, Wertschöpfung und Gerechtigkeit im Datenkapitalismus. Berlin: Econ.

Rotherham, Andrew J., und Daniel T. Willingham. 2010. «21st-Century〉 Skills: Not New, but a Worthy Challenge». American Educator 34 (1): 17-20.

Ryberg, Thomas, Jacob Davidsen, und Vivien Hodgson. 2018. «Understanding Nomadic Collaborative Learning Groups». British Journal of Educational TechnologY 49 (2): 235-47. https://doi.org/10.1111/bjet.12584.

Salomon, G., und Richard E. Clark. 1977. «Reexamining the methodology of research on media and technology in education». Review of educational research, 99-120.

Salomon, Gavriel. 1972. "Can we affect cognitive skills through visual media? An hypothesis and initial findings». Educational Technology Research and Development 20 (4): 401-22.

Schmidt, Siegfried J. 1996. Kognitive Autonomie und soziale Orientierung. Frankfurt am Main: Suhrkamp.

Schmidt, Siegfried J. 2003. Geschichten \& Diskurse: Abschied vom Konstruktivismus. Reinbek: Rowohlt Taschenbuch Verlag.

Siever, Torsten, Peter Schlobinski, und Jens Runkehl, Hrsg. 2009. Websprache.net: Sprache und Kommunikation im Internet. Walter de Gruyter.

Skinner, B.F. 1958. «Teaching machines». Science 128: 969-77.

Spitzer, M. 2005. Vorsicht Bildschirm! Elektronische Medien, Gehirnentwicklung, Gesundheit und Gesellschaft. Stuttgart: Klett.

Stalder, Felix. 2016. Kultur der Digitalität. Berlin: Suhrkamp Verlag.

Tamim, Rana M., Robert M. Bernard, Eugene Borokhovski, Philip C. Abrami, und Richard F. Schmid. 2011. "What Forty Years of Research Says About the Impact of Technology on Learning». Review of Educational Research 81 (1): 4-28.

Trilling, Bernie, und Charles Fadel. 2009. 21st Century Skills: Learning for Life in Our Times. 1. Aufl. San Francisco: Jossey-Bass.

Tulodziecki, Gerhard. 2015. «Dimensionen von Medienbildung. Ein konzeptioneller Rahmen für medienpädagogisches Handeln». MedienPädagogik. Zeitschrift für Theorie und Praxis der Medienbildung. 2015 (Occasional Papers), 31-49. https://doi.org/10.21240/mpaed/00/2015.06.05.X. 
Voogt, J., O. Erstad, C. Dede, und P. Mishra. 2013. "Challenges to Learning and Schooling in the Digital Networked World of the 21st Century». Journal of Computer Assisted Learning 29 (5): 403-13. https://doi.org/10.1111/jcal.12029.

West, Sarah Myers. 2019. «Data Capitalism: Redefining the Logics of Surveillance and Privacy». Business \& Society 58 (1): 20-4l. https://doi. org/10.1177/0007650317718185.

Wunder, Maik. 2018. Diskursive Praxis der Legitimierung und Delegitimierung von digitalen Bildungsmedien - Eine Diskursanalyse. Bad Heilbronn: Julius Klinkhardt.

Zierer, Klaus. 2019. Putting Learning Before Technology! The Possibilities and Limits of Digitalization. London; New York: Taylor \& Francis Ltd.

Zuboff, Shoshana. 2015. «Big Other: Surveillance Capitalism and the Prospects of an Information Civilization». Journal of Information Technology 30 (1): 75-89. https://doi.org/10.1057/jit.2015.5. 